\title{
Superferromagnetic domain state of a discontinuous metal insulator multilayer
}

\author{
S. Bedanta, ${ }^{1, *}$ O. Petracic, ${ }^{1}$ E. Kentzinger, ${ }^{2}$ W. Kleemann, ${ }^{1}$ U. Rücker, ${ }^{2}$ A. Paul, ${ }^{2}$ Th. Brückel, ${ }^{2}$ S. Cardoso,${ }^{3}$ and \\ P. P. Freitas ${ }^{3}$ \\ ${ }^{1}$ Angewandte Physik, Universität Duisburg-Essen, D-47048 Duisburg, Germany \\ ${ }^{2}$ Institut für Festkörperforschung, Forschungszentrum Jülich, D-52425 Jülich, Germany \\ ${ }^{3}$ INESC, Rua Alves Redol 9-1, 1000 Lisbon, Portugal
}

(Received 22 February 2005; published 12 July 2005)

\begin{abstract}
Polarized neutron reflectivity (PNR) and magnetometry studies have been performed on the granular multilayer $\left[\mathrm{Co}_{80} \mathrm{Fe}_{20}(1.3 \mathrm{~nm}) / \mathrm{Al}_{2} \mathrm{O}_{3}(3 \mathrm{~nm})\right]_{10}$. Due to strong interparticle interactions, a collective superferromagnetic state is encountered. Cole-Cole plots drawn from the complex ac susceptibility are measured as functions of frequency, temperature, and field amplitudes that hint at the relaxation, creep, sliding, and switching regimes of pinned domain walls that are in close agreement with results obtained from simulations. Very slow switching with exponential relaxation under near-coercive fields is confirmed by PNR measurements. The complete absence of spin-flip scattering confirms that the magnetization reversal is achieved merely by domain nucleation and growth.
\end{abstract}

DOI: 10.1103/PhysRevB.72.024419

PACS number(s): 75.40.Gb, 75.60.Ej, 61.10.Kw, 61.12.Ha

\section{INTRODUCTION}

Granular thin films, in which magnetic single-domain nanoparticles ("superspins") are dispersed in a nonmagnetic matrix, are a very active current research topic, because their magnetic and magnetotransport properties suggest various technological applications. ${ }^{1}$ They are represented, e.g., by discontinuous metal-insulator multilayers (DMIMs) that resemble frozen ferrofluids to a large extent. ${ }^{2,3}$ Due to magnetic interparticle interactions, e.g., of dipolar or tunneling exchange origin, different kinds of collective behavior are encountered, viz. modified superparamagnetism, ${ }^{4}$ superspin glass (SSG) behavior, ${ }^{5}$ or superferromagnetism (SFM). ${ }^{6,7}$ DMIMs have the advantage that their type of behavior can effectively be controlled by the nominal thickness of the deposited ferromagnetic (FM) material. Recently, we presented experimental evidence for SFM behavior in the DMIM $\left[\mathrm{Co}_{80} \mathrm{Fe}_{20}\left(t_{n}\right) / \mathrm{Al}_{2} \mathrm{O}_{3}(3 \mathrm{~nm})\right]_{10}$ with nominal thickness of the FM layers $t_{n}=1.4 \mathrm{~nm} .{ }^{7,8}$ This value lies below the structural percolation limit, $t_{p}=1.8 \mathrm{~nm}$, above which a conventional three-dimensional (3D) ferromagnetic FM phase with Ohmic conduction is encountered. ${ }^{3}$ Similar, though only twodimensional (2D) SFM states are found, e.g., in $\mathrm{Co}\left(t_{n}\right) / \mathrm{Cu}(100)$ thin films at coverages $t_{n}<1.8$ monolayers ${ }^{9}$ or in ultrathin Fe films grown on $\mathrm{CaF}_{2} / \mathrm{Si}(111) .{ }^{10}$

In several cases, the ferromagnetic interparticlecorrelations could be imaged, e.g., by small-angle neutron scattering in nonpercolated $\mathrm{Co}-\mathrm{SiO}_{2}$ granular films ${ }^{11}$ or by magnetic force microscopy (MFM) in self-assembled $\epsilon$-Co nanoparticle layers. ${ }^{12}$ However, in DMIMs the conjectured domain structure ${ }^{8}$ has not yet been evidenced in a direct way. A further step toward this aim is presented in this paper. Here we discuss data on the magnetic hysteresis, the complex $a c$ susceptibility, and polarized neutron reflectivity (PNR) of the SFM system $\left[\mathrm{Co}_{80} \mathrm{Fe}_{20}\left(t_{n}=1.3 \mathrm{~nm}\right) / \mathrm{Al}_{2} \mathrm{O}_{3}(3 \mathrm{~nm})\right]_{10}$. The complex ac susceptibility, $\chi^{\prime}-i \chi^{\prime \prime}$, as well as the time dependence of the neutron reflectivities reveal that the magnetic dynamic behavior can be explained within the concept of domain wall motion in an impure ferromagnet. ${ }^{8,13}$ It signifies that the granular systems behave like a thin FM film, only with the difference that the atomic moments have to be replaced by the superspin moments of the individual particles. While the FM nanoparticles remain single-domained, the ensemble shows collective SFM behavior. This idea is corroborated in particular by Cole-Cole plots, $\chi^{\prime \prime}$ vs. $\chi^{\prime} \cdot{ }^{14}$

\section{EXPERIMENTAL PROCEDURE}

DMIMs of $\left[\mathrm{Co}_{80} \mathrm{Fe}_{20}\left(t_{n}\right) / \mathrm{Al}_{2} \mathrm{O}_{3}(3 \mathrm{~nm})\right]_{10}$ with $t_{n}$ $=1.3 \mathrm{~nm}$ were prepared by Xe-ion beam sputtering on glass substrates. ${ }^{3}$ During the growth of the sample, a magnetic field of $\mu_{0} H \approx 10 \mathrm{mT}$ was applied parallel to the sample plane, which leads to an in-plane easy axis in the sample. High-resolution transmission electron micrographs (HRTEM) of a related sample with $t_{n}=0.9 \mathrm{~nm}^{15}$ have shown that nearly spherical CoFe granules are embedded in the amorphous $\mathrm{Al}_{2} \mathrm{O}_{3}$ matrix. One should note that our $t_{n}$ $=1.3 \mathrm{~nm}$ sample is from a new batch and compares roughly to a $t_{n}=1.45 \mathrm{~nm}$ sample from a previous set. ${ }^{7,8}$ Magnetization hysteresis and $a c$ susceptibility were measured by use of a superconducting quantum interference device (SQUID) magnetometer (MPMS-5S, Quantum Design). The ac susceptibility measurements were performed after setting flux gate controlled zero-field conditions to within $\left|\mu_{0} H\right|<2 \mu \mathrm{T}$ by quenching the superconducting solenoid and compensating its remanent field. A high-temperature option of the magnetometer (QD-M102, Quantum Design) enables us to perform measurements up to $800 \mathrm{~K}$ using a specially designed sample holder. ${ }^{16}$ The microstructure and the layer quality were investigated by $\mathrm{x}$-ray diffraction (XRD) and diffuse $\mathrm{X}$-ray scattering under grazing incidence using a Bruker-AXS D8 diffractometer with $\mathrm{Cu} \mathrm{K} \alpha(\lambda=0.154 \mathrm{~nm})$ radiation equipped with Göbel mirrors for the incident and reflected beams.

PNR under small angles has been performed with the HADAS reflectometer at the Jülich research reactor 


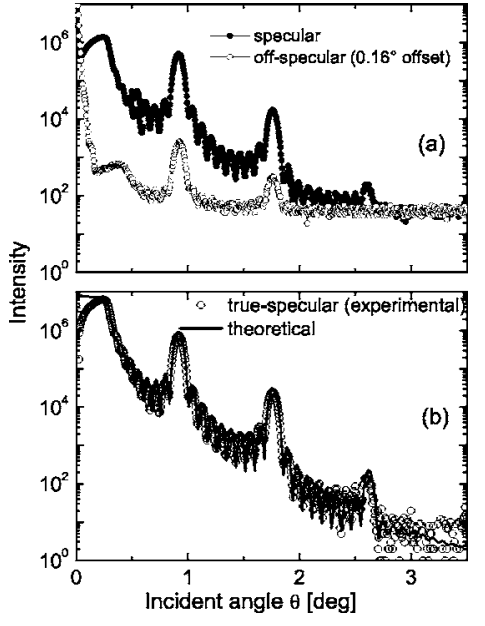

FIG. 1. (a) X-ray specular reflectivity and longitudinal diffuse scattering $\left(\theta-2 \theta+0.16^{\circ}\right)$ of a $\left[\mathrm{CoFe}(1.3 \mathrm{~nm}) / \mathrm{Al}_{2} \mathrm{O}_{3}(3 \mathrm{~nm})\right]_{10}$ multilayer. (b) The true-specular scan along with the best simulated curve for the multilayer is shown as a function of the angle of incidence.

FRJ-2 (DIDO). ${ }^{17}$ The main feature of this instrument is that it permits a simultaneous polarization analysis over the entire range of scattering angles $\left(\theta \leqslant 3^{\circ}\right)$. The magnetic field was provided by a pair of Helmholtz coils. It is homogeneous at the sample position and special care was taken to maintain the initial polarization of the neutron beam.

\section{RESULTS AND DISCUSSIONS}

\section{A. X-ray diffraction}

XRD scans under grazing incidence were taken to measure the specular and the diffuse scattered intensities. In specular reflectivity measurements, the momentum transfer is perpendicular to the sample surface, $q=q_{z}$. This type of measurement provides information about the individual layer thicknesses and an estimate of the lateral averaged (rms) roughness of the layers. The scattered intensity is measured both in the specular $(\theta-2 \theta)$ and in the longitudinal-diffuse $\left(\theta-2 \theta+0.16^{\circ}\right)$ geometry as shown in Fig. 1(a). The longitudinal-diffuse scattering peaks at the Bragg peak positions indicate a good vertical correlation in the multilayer stack.

Figure 1(b) shows the true-specular (specular minus offspecular) reflectivity curves along with their fit. One finds well-defined Bragg peaks up to the third order, and the presence of well-defined finite-thickness higher-frequency oscillations (Kiessig fringes) clearly indicates the presence of ten uniform bilayers. Simulations of the true-specular curve (solid line) yield $1.32 \pm 0.02 \mathrm{~nm}$ and $3.66 \pm 0.02 \mathrm{~nm}$ for the thicknesses of the $\mathrm{FeCo}$ and $\mathrm{Al}_{2} \mathrm{O}_{3}$ layers, respectively, with a rms roughness of $0.56 \pm 0.02 \mathrm{~nm}$ for both $\mathrm{Al}_{2} \mathrm{O}_{3}$ and $\mathrm{CoFe}$.

\section{B. Magnetization and $a c$ susceptibility}

In Fig. 2, the $d c$ magnetization curves $M$ vs the magnetic field $\mu_{0} H$ are shown at different temperatures, $T$, between 150 and $520 \mathrm{~K}$. Both the coercive field, $H_{c}$, and remanence,

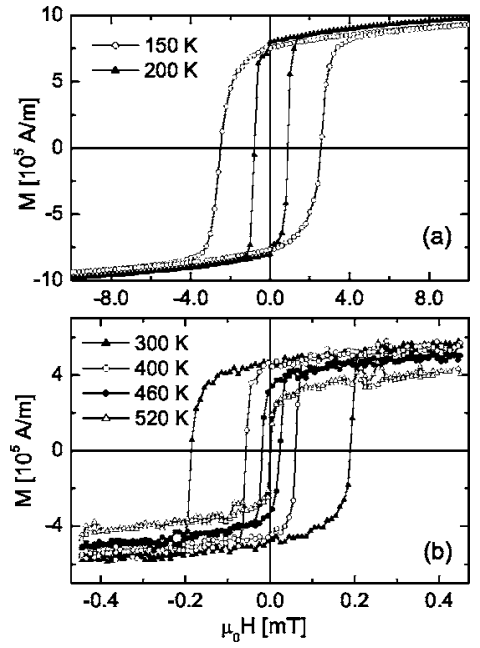

FIG. 2. (a) Magnetization vs applied field at $T=150$ and $200 \mathrm{~K}$ and (b) at 300, 400, 460, and $520 \mathrm{~K}$. The magnetization curves shown here are measured on two different pieces of the same sample.

$M_{r}$, decrease monotonically as $T$ increases, the latter reaching zero at $T_{c} \approx 510 \mathrm{~K}$. Rounded hysteresis loops indicate soft ferromagnetism, which partly demagnetizes in zero field via domain formation as in permalloy or $\mu$ metal. The thermoremanent magnetization $M^{T R M}$ shown in Fig. 3 is recorded after field cooling (FC) in $0.44 \mathrm{mT}$ from 520 to 380 $\mathrm{K}$, then switching off the field and measuring upon heating. Because $M^{T R M}(T)$ qualitatively reflects the behavior of the ferromagnetic order parameter, the curve hints at a critical temperature, $T_{c} \approx 510 \mathrm{~K}$, confirming the previous result from $M$ vs $H$ measurements (Fig. 2). The data shown here have been corrected for the diamagnetic moment of the sample holder and a systematic vertical shift being probably an artifact of the thermal expansion of our sample holder. ${ }^{16}$

Figure 4 shows the $a c$ susceptibility components $\chi^{\prime}$ and $\chi^{\prime \prime}$ vs $T$ taken with amplitudes $\mu_{0} H_{0}=0.4 \mathrm{mT}$ at frequencies $0.2 \leqslant f \leqslant 10^{3} \mathrm{~Hz}$. In the real part [Fig. 4(a)], one finds virtually no response at low temperatures. Only above a characteristic temperature, $T_{w}=T_{w}(f), \chi^{\prime}$ strongly increases. Interestingly, near $T_{w}$ an undershooting of the signal to negative values is observed. This behavior reflects the dynamic transition region between flat minor hysteresis and high switching loops, where parts of the loop show an increase of the magnetization, while the field is already decreasing and vice

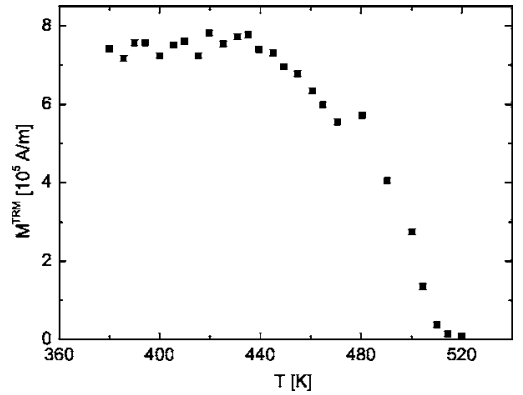

FIG. 3. Thermoremanent magnetization $\left(M^{T R M}\right)$ measured after field cooling in $\mu_{0} H=0.44 \mathrm{mT}$ vs temperature. 

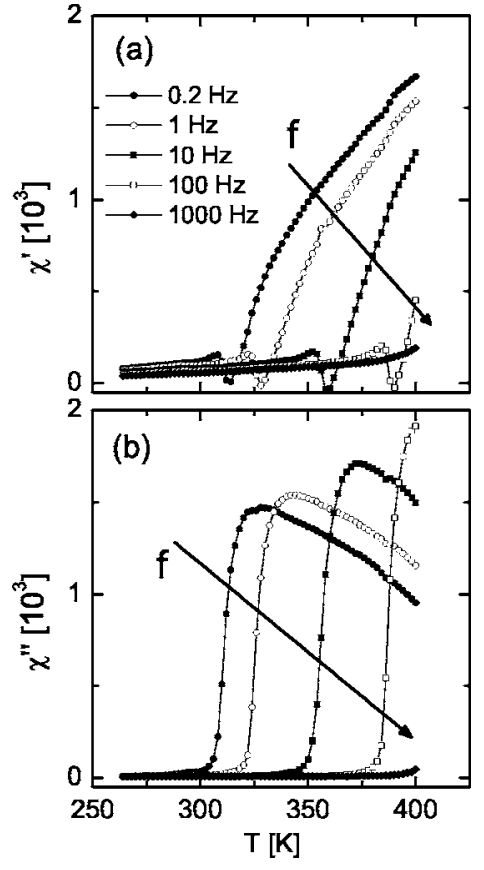

FIG. 4. $\chi^{\prime}$ (a) and $\chi^{\prime \prime}$ (b) vs $T$ measured at $a c$ amplitudes $\mu_{0} H$ $=0.4 \mathrm{mT}$ and frequencies $200 \mathrm{mHz} \leqslant f \leqslant 1 \mathrm{kHz}$.

versa. This corresponds to the observed negative susceptibility contributions. Hence, the total $\chi^{\prime}$ signal is reduced or even negative. The imaginary part [Fig. 4(b)] shows a maximum near $T_{w}$ corresponding to the largest area of the dynamic hysteresis loop and to the point of inflexion of the real part. Plotting these data as frequency spectra (Fig. 5) reveals a behavior close to that found from simulations of a field-

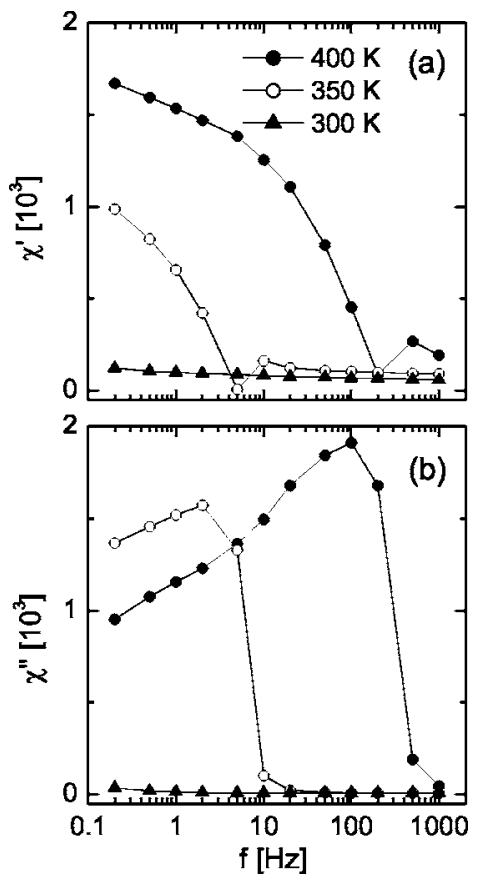

FIG. 5. $\chi^{\prime}$ (a) and $\chi^{\prime \prime}$ (b) vs frequency measured at an $a c$ amplitude $\mu_{0} H=0.4 \mathrm{mT}$ at $T=300,350$, and $400 \mathrm{~K}$. Data points are connected by lines.

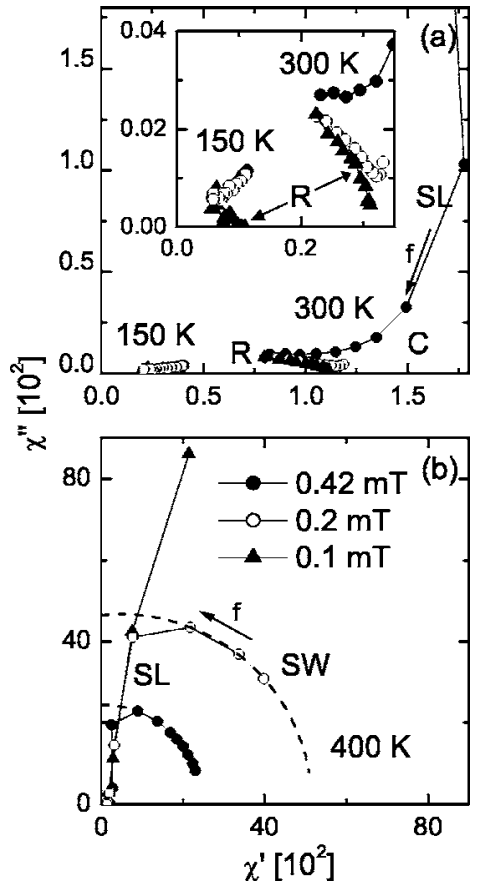

FIG. 6. Cole-Cole plots $\chi^{\prime \prime}$ vs $\chi^{\prime}$ measured at ac amplitudes $\mu_{0} H=0.1,0.2$, and $0.42 \mathrm{mT}$ at $10 \mathrm{mHz} \leqslant f \leqslant 1 \mathrm{kHz}$ (order indicated by arrows) at $T=150$ and $300 \mathrm{~K}$ (a) and $400 \mathrm{~K}$ (b). Data points are connected by solid lines. The data referring to 0.2 and $0.42 \mathrm{mT}$ in (b) are fitted to quarter circles as represented by dashed lines. Dynamic regimes referring to relaxation, creep, sliding, and switching are marked by R, C, SL, and SW, respectively.

driven domain wall (DW) in a random medium. ${ }^{13}$ Here the real part $\chi^{\prime}$ shows a sharply rising response below a certain threshold frequency $f_{c}=f_{c}(T)$, while the imaginary part $\chi^{\prime \prime}$ exhibits a peak near to $f_{c}$.

The spectra can also be presented as Cole-Cole plots, $\chi^{\prime \prime}$ vs $\chi^{\prime},{ }^{14}$ which were shown previously to be a more adequate and unequivocal method of characterizing the dynamical behavior of randomly pinned DW systems. ${ }^{8,13}$ Figure 6 shows Cole-Cole plots at amplitudes $\mu_{0} H_{0}=0.1,0.2$, and $0.42 \mathrm{mT}$ of the ac susceptibility data taken at three different temperatures, $T=150,300$, and $400 \mathrm{~K}$. We are able to observe four distinct dynamic regions. (i) Relaxation, as seen from the flattened partial semicircles at $T=150$ and $300 \mathrm{~K}$ in Fig. 6(a) for high frequencies designated as "R." It should be noticed that in this context the term "relaxation" means a kinetic state of motion, where the external field is not able to displace the center of gravity of the DWs, but merely gives rise to local hopping between adjacent free energy double wells. This mode is absent in dc field excitations. (ii) Creep, as found from the linear increase at $300 \mathrm{~K}$ in Fig. 6(a) is designated as "C." This region refers to thermally activated nonadiabatic motion of a DW. (iii) Slide, from the almost vertical parts observed at $400 \mathrm{~K}$ in Fig. 6(b) is designated as "SL." Slide is also known as the adiabatic viscous motion of the DW. Finally, (iv) switching, as seen from the quarter circles in Fig. 6(b) is designated as "SW". Here the magnetization is flipped from negative to positive saturation and vice versa. Region (i) is well understood in ferroelectric systems, ${ }^{18}$ where the DW shows a polydispersive response, 
while no net movement of the center of gravity of the DW is encountered. This occurs for small field amplitudes and/or high frequencies, which do not allow the DW to be released from local pinning energy potentials. Upon increase of the field amplitude, the DW is locally depinned and enters the creep regime, which is clearly seen in Fig. 6(a) for $\mu_{0} H_{0}$ $=0.42 \mathrm{mT}$. Due to our technical limitation of $\mu_{0} H_{0}$ to 0.45 $\mathrm{mT}$, the only possibility to enter both the slide and switching regimes is to increase the temperature, e.g., to $T=400 \mathrm{~K}$, Fig. 6(b).

The dynamic response in the four regions can be described phenomenologically by two expressions of the complex susceptibility. The creep and slide regimes obey inverse power laws

$$
\chi(\omega)=\chi_{\infty}\left[1+(i \omega \tau)^{-\beta}\right]
$$

with an asymptotic value $\chi_{\infty}$, an effective relaxation time $\tau$, and an exponent $0<\beta \leqslant 1,8,18,19$ This yields the observed linear relationship $\chi^{\prime \prime}\left(\chi^{\prime}\right)=\left(\chi^{\prime}-\chi_{\infty}\right) \tan (\pi \beta / 2)$. In the creep region, one finds a finite slope in the $\chi^{\prime \prime}\left(\chi^{\prime}\right)$ data implying that $\beta<1$. The sliding regime has asymptotically a purely imaginary response $\chi^{\prime \prime}$ and can be described by $\beta=1 .{ }^{8}$ Both types of behavior correspond well to the results obtained from simulations of a DW in an impure FM, where $\beta=1$ in the case of an adiabatic and $\beta<1$ in the case of a nonadiabatic motion of the DW. ${ }^{13}$

Both the relaxation and switching regimes are well characterized by a Cole-Cole type expression ${ }^{14}$

$$
\chi(\omega)=\chi_{\infty}+\frac{\chi_{0}-\chi_{\infty}}{1+(i \omega \tau)^{1-\alpha}},
$$

where the exponent $\alpha$ with $0 \leqslant \alpha<1$ is again a phenomenological exponent reflecting the polydispersity of the system. The case $\alpha=0$ yields the standard Debye-type relaxator with one single relaxation time. This is applicable to the switching regime, where the dynamic behavior of the system can be understood as a monodisperse relaxation process. The relaxation time $\tau$ is characterized by the total time of motion of (in the simplest case) one DW across the sample during half a field cycle. For values of $\omega=2 \pi f$ larger than $1 / \tau$, the halfperiod of the field cycle becomes smaller than the intrinsic "relaxation time" of the switching, i.e., the time the DW needs to move from one side to the other. Hence, in this frequency regime, the system does not switch and enters the slide region where both $\chi^{\prime}$ and $\chi^{\prime \prime}$ rapidly decrease as $\omega$ increases [SL regions in Fig. 6(b)]. However, for $\omega<1 / \tau$ complete hysteresis loops are traveled through. They become narrower and higher as $\omega$ decreases. This is described by the right half of the Debye semicircles [SW regions in Fig. 6(b)], whose apexes correspond to $\omega=1 / \tau$.

In contrast to switching, the relaxation regime is described by Eq. (2) using $\alpha \neq 0$, which yields a flattened semicircle in the Cole-Cole presentation. ${ }^{14}$ This corresponds well to the result found in the experiment [Fig. 6(a)], where data referring to the decreasing low- $f$ branches of the flattened circles are observed at 150 and $300 \mathrm{~K}$. One should note that the crossovers relaxation-to-creep, creep-to-slide, and slideto-switch as found in Figs. 6(a) and 6(b) are necessarily smeared, because the applied field during the cycle naturally covers different field values and hence mixes the different modes of motion.

\section{Polarized neutron reflectivity}

In a PNR experiment, the intensity of the neutrons reflected from a surface is measured as a function of the component of the momentum transfer that is perpendicular to the surface, $q_{z}=4 \pi \sin \theta / \lambda$, where $\theta$ is the angle of incidence (and reflection) and $\lambda$ is the neutron wavelength. Because $q_{z}$ is a variable conjugate to the depth $z$ from the surface of the film, a scan over a suitable range of $q_{z}$ provides excellent information on the magnetic depth profile of the film.

Additional information, like the separation of structural and magnetic densities as well as the magnetization directions, can be obtained from polarization analysis, where the polarization of the incident neutron beam is prepared in a definite state + or - for polarization parallel or antiparallel to the applied external field, respectively, and the final polarization is being measured. Thus four different cross sections are obtained, which are conventionally designated as $R^{++}, R^{--}, R^{+-}$, and $R^{-+}$. The non-spin-flip (NSF) data, $R^{++}$ and $R^{--}$, depend on the chemical structure, as well as on the projection of the in-plane magnetization parallel to the applied field. The spin-flip (SF) cross sections, $R^{+-}$and $R^{-+}$, arise solely from the projection of the in-plane magnetization perpendicular to the applied field. ${ }^{20}$

Our PNR measurements were performed at $150 \mathrm{~K}$ with polarization analysis simultaneously over the whole range $\left(\theta \approx 3^{\circ}\right)$ of scattering angles. At this temperature, the coercive field is $\mu_{0} H_{c} \approx 2.5 \mathrm{mT}$ and the sample almost saturates at $10 \mathrm{mT}$ [Fig. 2(a)]. The wavelength of the neutron beam is $0.452 \mathrm{~nm}$ and the magnetic field is always applied parallel to the sample plane and to the easy axis.

The PNR data shown in Fig. 7(a) were taken at positive saturation $(12 \mathrm{mT})$. The FM state of the sample can be recognized by the splitting of the reflectivity edges where $R^{++}(\theta)$ is shifted to higher angles in comparison to $R^{--}(\theta)$. This can be explained as follows. The critical scattering vectors $Q_{c}$, below which the neutrons are totally reflected, are different for the $(++)$ and $(--)$ states according to ${ }^{21}$

$$
Q_{c}=[16 \pi N(b \pm p \sin \phi)]^{1 / 2},
$$

where $b$ is the nuclear and $p$ the magnetic scattering lengths and $N$ is the atomic or nuclear number density. For a nonmagnetic matrix, $Q_{c}$ is $(16 \pi N b)^{1 / 2}$. $\phi$ is the angle between the direction of magnetization of the sample to the SF axis as shown in the inset of Fig. 7(b). ${ }^{21}$ When the magnetization in the sample is parallel to the guiding field, corresponding to $\phi=90^{\circ}$, we arrive at $Q_{c}^{++}=[16 \pi N(b+p)]^{1 / 2}$ and $Q_{c}^{--}$ $=[16 \pi N(b-p)]^{1 / 2}$, respectively. Inserting the nuclear scattering lengths of $\mathrm{CoFe}$ and $\mathrm{Al}_{2} \mathrm{O}_{3}$, i.e., 4.4 and $5.1 \mathrm{fm}$, respectively, and the magnetic scattering length of $\mathrm{CoFe}$, i.e., 1.7 $\mathrm{fm}$, one obtains $Q_{c}^{++}>Q_{c}^{--}$[see arrows in Fig. 7(a)].

For the superlattice Bragg peak, the contrast is determined by the difference between $(b+p)$ and $(b-p)$ of CoFe compared to $b$ of $\mathrm{Al}_{2} \mathrm{O}_{3}$. Hence in the positively saturated state, the superlattice Bragg peak occurs in the $R^{--}$channel. This is 


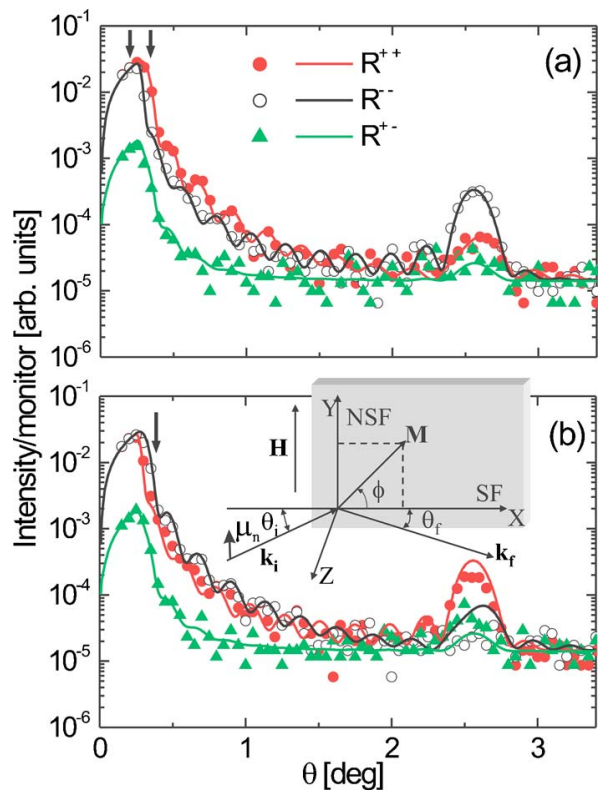

FIG. 7. (Color online) PNR reflectivities $R^{++}$in red (dark gray), $R^{--}$in black, and $R^{+-}$in green (light gray) vs $\theta$ measured at $T$ $=150 \mathrm{~K}$ and $\mu_{0} H=12 \mathrm{mT}$ (saturation) (a) and close to remanence at the guiding field of the neutrons, $\mu_{0} H=0.94 \mathrm{mT}$, after negative saturation (b). The limits of total reflection, $Q_{c}^{++}$and $Q_{c}^{--}$are designated by vertical arrows in (a). The vertical arrow at $\theta=0.35^{\circ}$ in (b) refers to the scattering data in Fig. 8. Error bars (not shown) are smaller than 0.001 a.u. and removed for better clarity.

seen in Fig. 7(a), where the first-order structural superlattice Bragg peak at $\theta=2.56^{\circ}$ is dominant in the $R^{--}$channel. Its position corresponds to a bilayer thickness $d \approx 5 \mathrm{~nm}$ in agreement with the $\mathrm{x}$-ray results shown in Fig. 1. The presence of a weak signal in the two SF channels, $R^{+-}$and $R^{-+}$, is primarily due to the finite-flipping ratio of 18 corresponding to a limited efficiency of $94 \%$ for the polarization analysis.

The PNR data shown in Fig. 7(b) were taken at a weak guiding field $(0.94 \mathrm{mT})$ of the neutrons after negatively saturating the sample. They correspond to the negative remanence as confirmed by the small-angle data close to the plateau of total reflection, e.g., at $\theta=0.35^{\circ}$ (arrow), where $R^{--}$ $>R^{++}$. The first-order structural superlattice peak at $\theta$ $=2.56^{\circ}$ is here dominant in the $R^{++}$channel, confirming that the net magnetization in the sample is antiparallel to the external field. As there is no significant SF scattering, there is obviously no magnetization component perpendicular to the applied field. It is worth mentioning that PNR measurements performed at various other points of the hysteresis loop yield no SF scattering, which hints at the absence of any in-plane transverse magnetization component in the sample.

This is corroborated by dynamic PNR measured upon field switching. Figures $8(a)$ and 8 (b) show the temporal relaxation of the neutron intensities $R=R^{++}-R^{--}$and $r=R^{+-}$ $+R^{-+}$at $\mu_{0} H=1.7 \mathrm{mT}$ and $1.9 \mathrm{mT}$ (close to the coercive field), respectively. The field is applied parallel to the sample plane after negative saturation and measured at $\theta=0.35^{\circ}$, where $R^{--}>R^{++}$[arrow in Fig. 7(b)]. Hence, at the beginning of the experiment, the net magnetization of the sample is antiparallel to the applied field, but an interchange of $R^{--}$

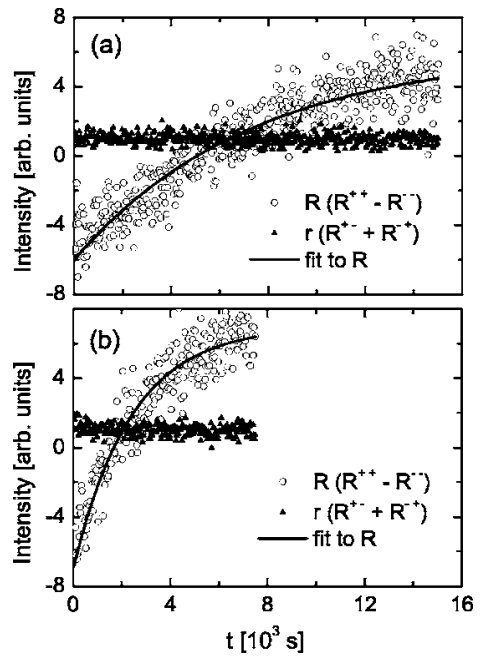

FIG. 8. Relaxation of the PNR components $R^{++}-R^{--}$(NSF) and $R^{+-}+R^{-+}(\mathrm{SF})$ vs time at $T=150 \mathrm{~K}$ and $\theta=0.35^{\circ}$ with a field $\mu_{0} H$ $=1.7 \mathrm{mT}$ (a) and $1.9 \mathrm{mT}$ (b) close to coercivity. Error bars (not shown) are smaller than 1 a.u. The solid lines represent best fits of Eq. (4) to the NSF intensities (see text).

and $R^{++}$occurs after about $4 \times 10^{3} \mathrm{~s}$ in Fig $8(\mathrm{a}),{ }^{23}$ and 2 $\times 10^{3} \mathrm{~s}$ in Fig $8(\mathrm{~b})$. The NSF intensities are satisfactorily fitted by exponential decay laws,

$$
R(t)=R_{\infty}[1-2 \exp (-t / \tau)]
$$

(solid lines) with $R_{\infty}=6.0 \pm 0.1, \tau=(7.2 \pm 0.1) \times 10^{3} \mathrm{~s}$ and $R_{\infty}$ $=7.0 \pm 0.1, \tau=(2.40 \pm 0.06) \times 10^{3} \mathrm{~s}$, respectively. On the other hand, there is no significant temporal change of $r$.

Within the Fatuzzo-Labrune model, ${ }^{22}$ the observed exponential magnetization reversal, Eq. (4), under near-coercive fields seems to indicate nucleation dominated aftereffects. This appears reasonable, because DWs in superferromagnets are presumed to cost merely a stray field, but no exchanged energy. However, the large error bars of the relaxation curves cannot exclude deviations toward nonexponential (e.g., logarithmic) behavior. It will be interesting to observe the reversal by appropriate methods in real space and to judge on the ratio between nucleation and DW propagation events.

In order to motivate the use of $R$ and $r$ instead of the original data, $R^{++}(t)$ etc., let us assume that the magnetization vector $\mathbf{M}$ lies in the sample plane with an angle $\phi$ with respect to the horizontal $x$ axis [inset of Fig. 7(b)]. Hence, it will be perpendicular to the scattering vector $\mathbf{Q}$. Next we consider $^{21}$ that a monochromatic and polarized neutron beam is incident onto the sample at a scattering angle $\theta$ and that the magnetic moments of the incoming monochromatic neutrons are aligned normal to the scattering plane and parallel to the sample surface, i.e., parallel to the transverse component of magnetization $M_{y}$. Let us define $V_{m}$ as the magnetic potential that the neutron experiences in the sample, which can be expressed as

$$
V_{m}=-\mu B_{e f f \|}=\left(2 \pi h^{2} / m\right) N_{A} p,
$$

where $\mu$ is the neutron magnetic moment, $B_{\text {effl }}$ the effective magnetic flux density in the sample plane, $m$ the neutron 
mass, $N_{A}$ the atomic density and $p$ the magnetic scattering length. Then the difference of the two non-spin-flip components

$$
R=2 p \sin \phi=2 p_{y} \propto M_{y},
$$

is proportional to the $y$ component of the magnetization $M_{y}$, whereas the SF reflectivities $R^{+-}=R^{-+}$are degenerate, and

$$
r=2 p \cos \phi \propto M_{x}^{2}
$$

is proportional to the square of the $x$ component $M_{x}$.

Figures 8(a) and 8(b) clearly show only changes in the NSF intensities, while the SF intensities are almost constant during the measurements. Therefore, one can conclude that only the longitudinal magnetization component parallel to the applied field is present in the sample, whereas the transverse magnetization component is negligible. This hints at the absence of magnetization rotation and corroborates the suspected DW nucleation and growth processes during switching near the coercive field. ${ }^{8}$ Similar PNR data were observed on thin films of $\mathrm{Fe}$ exchange coupled to antiferromagnetic $\mathrm{FeF}_{2},{ }^{24}$ where a field close to the coercive field was applied parallel to the sample plane. The absence of SF signals during magnetization reversal was attributed to mere nucleation and growth processes of magnetic domains, which also applies to our SFM sample.
Unfortunately we did not observe any off-specular scattering, from which one could calculate the mean domain size in the sample. This might be due either to the limited scattering intensity or to the resolution limit of this PNR experiment, which cannot resolve domains being smaller than $\xi$ $\approx 0.5 \mu \mathrm{m}$.

\section{CONCLUSION}

The $a c$ susceptibility measured on the granular SFM multilayer $\left[\mathrm{Co}_{80} \mathrm{Fe}_{20}(1.3 \mathrm{~nm}) / \mathrm{Al}_{2} \mathrm{O}_{3}(3 \mathrm{~nm})\right]_{10}$ reveals signatures of all four possible dynamical regimes of DW motion in a random magnet, viz. relaxation, creep, slide, and switching. The PNR measurements evidence the absence of transverse magnetization components under static and dynamic conditions. The slow relaxation of PNR intensities shows that the magnetization reversal occurs through viscous DW motion. Experimentally it still remains a challenge to image the DWs and their field-induced displacement, e.g., by scanning MFM.

\section{ACKNOWLEDGMENTS}

We thank X. Chen for valuable discussions and the DFG (Graduate School "Structure and Dynamics of Heterogeneous Systems") for financial support.
*Corresponding author. Email address: sbedanta@kleemann.uniduisburg.de

${ }^{1}$ G. A. Prinz, Science 282, 1660 (1998); G. A. Prinz and J. H. Hathaway, Phys. Today 48 (4), 24 (1995); P. Grünberg, ibid. 54, 31 (2001).

${ }^{2}$ B. Dieny, S. Sankar, M. R. McCartney, D. J. Smith, P. BayleGuillemaud, and A. E. Berkowitz, J. Magn. Magn. Mater. 185, 283 (1998)

${ }^{3}$ G. N. Kakazei, Yu. G. Pogorelov, A. M. L. Lopes, J. B. Sousa, S. Cardoso, and P. P. Freitas, M. M. Pereira de Azevedo, and E. Snoeck, J. Appl. Phys. 90, 4044 (2001).

${ }^{4}$ J. L. Dormann, D. Fiorani, and E. Tronc, Adv. Chem. Phys. 98, 283 (1997)

${ }^{5}$ C. Djurberg, P. Svedlindh, P. Nordblad, M. F. Hansen, F. Bodker, and S. Morup, Phys. Rev. Lett. 79, 5154 (1997).

${ }^{6}$ S. Morup, M. B. Madsen, J. Franck, J. Villadsen, and C. J. W. Koch, J. Magn. Magn. Mater. 40, 163 (1983).

${ }^{7}$ W. Kleemann, O. Petracic, Ch. Binek, G. N. Kakazei, Y. G. Pogorelov, J. B. Sousa, S. Cardoso, and P. P. Freitas, Phys. Rev. B 63, 134423 (2001).

${ }^{8}$ X. Chen, O. Sichelschmidt, W. Kleemann, O. Petracic, Ch. Binek, J. B. Sousa, S. Cardoso, and P. P. Freitas, Phys. Rev. Lett. 89, 137203 (2002).

${ }^{9}$ U. Bovensiepen, P. Poulopoulos, W. Platow, M. Farle, and K. Baberschke, J. Magn. Magn. Mater. 192, L386 (1999).

${ }^{10}$ M. R. Scheinfein, K. E. Schmidt, K. R. Heim, and G. G. Hembree, Phys. Rev. Lett. 76, 1541 (1996).

${ }^{11}$ S. Sankar, A. E. Berkowitz, D. Dender, J. A. Borchers, R. W. Erwin, S. R. Kline, and David J. Smith, J. Magn. Magn. Mater.
221, 1 (2000).

${ }^{12}$ V. F. Puntes, P. Gorostiza, D. M. Aruguete, N. G. Bastus, and A. P. Alivisatos, Nat. Mater. 3, 263 (2004).

${ }^{13}$ O. Petracic, A. Glatz, and W. Kleemann, Phys. Rev. B 70, 214432 (2004); O. Petracic, X. Chen, O. Sichelschmidt, Ch. Binek, W. Kleemann, A. Glatz, T. Nattermann, S. Cardoso, and P. P. Freitas, J. Magn. Magn. Mater. 272-276, E1201 (2004).

${ }^{14}$ K. S. Cole and R. H. Cole, J. Chem. Phys. 9, 341 (1941); A. K. Jonscher, Dielectric Relaxation in Solids (Chelsea Dielectrics Press, London, 1983).

${ }^{15}$ S. Sahoo, O. Petracic, W. Kleemann, S. Stappert, G. Dumpich, P. Nordblad, S. Cardoso, and P. P. Freitas, Appl. Phys. Lett. 82, 4116 (2003).

${ }^{16}$ S. Bedanta, O. Petracic, M. Aderholz, and W. Kleemann, Rev. Sci. Instr. (2005) in print.

${ }^{17}$ U. Rücker, B. Alefeld, W. Bergs, E. Kentzinger, and Th. Brückel, Physica B 276-278, 95 (2000); U. Rücker, W. Bergs, B. Alefeld, E. Kentzinger, and Th. Brückel, Physica B 297, 140 (2001).

${ }^{18}$ W. Kleemann, J. Dec, S. Miga, Th. Woike, and R. Pankrath, Phys. Rev. B 65, 220101(R) (2002); Th. Braun, W. Kleemann, J. Dec, and P. A. Thomas, Phys. Rev. Lett. 94, 117601 (2005).

${ }^{19}$ A. A. Fedorenko, V. Mueller, and S. Stepanow, Phys. Rev. B 70, 224104 (2004).

${ }^{20}$ H. Zabel, Physica B 198, 156 (1994).

${ }^{21}$ H. Zabel, and K. Theis-Bröhl, J. Phys.: Condens. Matter 15, S505 (2003).

${ }^{22}$ E. Fatuzzo, Phys. Rev. 127, 1999 (1962); M. Labrune, S. Andrieu, F. Rio, and P. Bernstein, J. Magn. Magn. Mater. 80, 211 
(1989).

${ }^{23}$ S. Bedanta, X. Chen, S. Sahoo, W. Kleemann, E. Kentzinger, P. Nordblad, S. Cardoso, and P. P. Freitas, Phys. Status Solidi C 1, 3288 (2004).
${ }^{24}$ M. R. Fitzsimmons, C. Leighton, J. Nogues, A. Hoffmann, K. Liu, C. F. Majkrzak, J. A. Dura, J. R. Groves, R. W. Springer, P. N. Arendt, V. Leiner, H. Lauter, and Ivan K. Schuller, Phys. Rev. B 65, 134436 (2002). 